\title{
JOINT VALUE-DISTRIBUTION THEOREMS ON LERCH ZETA-FUNCTIONS. II
}

\author{
A. Laurinčikas \\ Faculty of Mathematics and Informatics, Vilnius University, Naugarduko 24, LT-03225 Vilnius \\ (e-mail: antanas.laurincikas@maf.vu.lt) \\ Kohji Matsumoto \\ Graduate School of Mathematics, Nagoya University, Chikusa-ku, Nagoya, 464-8602, Japan \\ (e-mail: kohjimat@math.nagoya-u.ac.jp)
}

\begin{abstract}
We give corrected statements of some theorems from [5] and [6] on joint value distribution of Lerch zeta-functions (limit theorems, universality, functional independence). We also present a new direct proof of a joint limit theorem in the space of analytic functions and an extension of a joint universality theorem.

Key words: Lerch zeta-function, limit theorem, probability measure, space of analytic functions, support, universality, weak convergence.
\end{abstract}

\section{INTRODUCTION}

Let, as usual, $s=\sigma+i t$, denote a complex variable, and let $\mathbb{N}, \mathbb{N}_{0}, \mathbb{Z}, \mathbb{R}$ and $\mathbb{C}$ be the set of all positive integers, non-negative integers, integers, real and complex numbers, respectively. The Lerch zeta-function $L(\lambda, \alpha, s)$ with fixed parameters $\alpha, \lambda \in \mathbb{R}, 0<\alpha \leqslant 1$, for $\sigma>1$, is defined by

$$
L(\lambda, \alpha, s)=\sum_{m=0}^{\infty} \frac{\mathrm{e}^{2 \pi i \lambda m}}{(m+\alpha)^{s}} .
$$

If $\lambda \in \mathbb{Z}$, then the Lerch zeta-function becomes the Hurwitz zeta-function

$$
\zeta(s, \alpha)=\sum_{m=0}^{\infty} \frac{1}{(m+\alpha)^{s}}, \quad \sigma>1,
$$

and $\zeta(s, \alpha)$, for $\alpha=1$, reduces to the Riemann zeta-function.

The paper is conditioned by [5] and [6], where the joint value distribution of Lerch zeta-functions was considered and some inaccuracies in the statements of some theorems in these papers were remained. The aim of this paper is to correct and comment the results of [5] and [6], and to give for some of them new proofs 
as well as certain their extensions. For this, first we recall the results of [5]. Denote by $\mathcal{B}(S)$ the class of Borel sets of the space $S$, and let, for $T>0$,

$$
\nu_{T}^{t}(\ldots)=\frac{1}{T} \operatorname{meas}\{t \in[0, T]: \ldots\}
$$

where meas $\{A\}$ is the Lebesgue measure of a measurable set $A \subset \mathbb{R}$, in place of dots a condition satisfied by $t$ is to be written, and the sign $t$ in $\nu_{T}^{t}$ means that the measure is taken over $t \in[0, T]$.

Let $r \in \mathbb{N} \backslash\{1\}$, and let $L\left(\lambda_{1}, \alpha_{1}, \sigma_{1}+i t\right), \ldots, L\left(\lambda_{r}, \alpha_{r}, \sigma_{r}+i t\right)$ be a collection of Lerch zeta-functions. Throughout the paper, as in [5], we suppose that $\lambda_{j} \notin \mathbb{Z}$, $j=1, \ldots, r$.

Theorem 1 of [5] remains without any changes. It asserts that, for $\min _{1 \leqslant j \leqslant r} \sigma_{j}>$ $\frac{1}{2}$, on $\left(\mathbb{C}^{r}, \mathcal{B}\left(\mathbb{C}^{r}\right)\right)$, there exists a probability measure $P$ such that the probability measure

$$
\nu_{T}^{t}\left(\left(L\left(\lambda_{1}, \alpha_{1}, \sigma_{1}+i t\right), \ldots, L\left(\lambda_{r}, \alpha_{r}, \sigma_{r}+i t\right)\right) \in A\right), \quad A \in \mathcal{B}\left(\mathbb{C}^{r}\right),
$$

converges weakly to $P$ as $T \rightarrow \infty$. Now we will present the corrected statement of Theorem 2 from [5]. This theorem deals with joint distribution of Lerch zetafunctions in the space of analytic functions, and differently from Theorem 1, gives the explicit form of the limit measure. For its statement, we need some additional notation. For $D=\left\{s \in \mathbb{C}: \sigma>\frac{1}{2}\right\}$, denote by $H(D)$ the space of analytic on $D$ functions equipped with the topology of uniform convergence on compacta, and let $H^{r}(D)=\underbrace{H(D) \times \ldots \times H(D)}_{r}$. Let $\gamma=\{s \in \mathbb{C}:|s|=1\}$, and define

$$
\Omega=\prod_{m=0}^{\infty} \gamma_{m}
$$

where $\gamma_{m}=\gamma$ for all $m \in \mathbb{N}_{0}$. By the Tikhonov theorem the infinite-dimensional torus $\Omega$ is a compact topological Abelian group, therefore, the probability Haar measure $m_{H}$ on $(\Omega, \mathcal{B}(\Omega))$ can be defined. This gives a probability space $\left(\Omega, \mathcal{B}(\Omega), m_{H}\right)$. Denote by $\omega(m)$ the projection of $\omega \in \Omega$ to the coordinate space $\gamma_{m}$. Moreover, define $\Omega^{r}=\Omega_{1} \times \ldots \times \Omega_{r}$, where $\Omega_{j}=\Omega$ for $j=1, \ldots, r$. Then $\Omega^{r}$ is also a compact topological group. Denote by $m_{H r}$ the probability Haar measure on $\left(\Omega^{r}, \mathcal{B}\left(\Omega^{r}\right)\right)$, and on the probability space $\left(\Omega^{r}, \mathcal{B}\left(\Omega^{r}\right), m_{H r}\right)$ define an $H^{r}(D)$-valued random element $\underline{L}(s, \underline{\omega})$ by

$$
\underline{L}(s, \underline{\omega})=\left(L\left(\lambda_{1}, \alpha_{1}, s, \omega_{1}\right), \ldots, L\left(\lambda_{r}, \alpha_{r}, s, \omega_{r}\right)\right),
$$

where

$$
L\left(\lambda_{j}, \alpha_{j}, s, \omega_{j}\right)=\sum_{m=0}^{\infty} \frac{\mathrm{e}^{2 \pi i \lambda_{j} m} \omega_{j}(m)}{\left(m+\alpha_{j}\right)^{s}},
$$


$\omega_{j} \in \Omega, j=1, \ldots, r$, and $\underline{\omega}=\left(\omega_{1}, \ldots, \omega_{r}\right)$. Denote by $P_{L}$ the distribution of the random element $\underline{L}(s, \underline{\omega})$, that is

$$
P_{\underline{L}}(A)=m_{H r}\left(\underline{\omega} \in \Omega^{r}: \underline{L}(s, \underline{\omega}) \in A\right), \quad A \in \mathcal{B}\left(H^{r}(D)\right),
$$

and define the probability measure $P_{T}$ by

$$
P_{T}(A)=\nu_{T}^{\tau}\left(\left(L\left(\lambda_{1}, \alpha_{1}, s+i \tau\right), \ldots, L\left(\lambda_{r}, \alpha_{r}, s+i \tau\right)\right) \in A\right), \quad A \in \mathcal{B}\left(H^{r}(D)\right) .
$$

We recall that the numbers $a_{1}, \ldots, a_{r}$ are algebraically independent over the field of rational numbers $\mathbb{Q}$ if the coefficients of every polynomial $p$ with rational coefficients satisfying $p\left(a_{1}, \ldots, a_{r}\right)=0$ are equal to zero.

THEOREM 1. Suppose that $\alpha_{1}, \ldots, \alpha_{r}$ are algebraically independent over $\mathbb{Q}$ and that $\lambda_{j} \notin \mathbb{Z}, j=1, \ldots, r$. Then the probability measure $P_{T}$ converges weakly to $P_{\underline{L}}$ as $T \rightarrow \infty$.

Theorem 1 is the corrected statement of Theorem 2 from [5]. In Theorem 2 of [5] it is required that the numbers $\alpha_{1}, \ldots, \alpha_{r}$ should be transcendental, however, this is not sufficient for the proof of Lemma 9 of [5]. Since, for $\sigma>1$, the shifting parameters of the functions $L\left(\lambda_{1}, \alpha_{1}, s\right), \ldots, L\left(\lambda_{r}, \alpha_{r}, s\right)$ are different, for the proof of Lemma 9 of [5] we need a limit theorem on the torus $\Omega^{r}$ (see Lemma 4 below). Moreover, in this theorem, the limit measure must be the Haar measure. Therefore, we have to use a condition that the set

$$
\bigcup_{j=1}^{r} \bigcup_{m=0}^{\infty}\left\{\log \left(m+\alpha_{j}\right)\right\}
$$

should be linearly independent over $\mathbb{Q}$. The latter requirement is satisfied if the numbers $\alpha_{1}, \ldots, \alpha_{r}$ are algebraically independent over $\mathbb{Q}$. This is also used in application of elements of ergodic theory for the identification of the limit measure. So, except for the above change in the proof of Lemma 9 of [5], the proof of Theorem 2 from [5] remains the same, and only in its statement the condition of the transcendence of $\alpha_{1}, \ldots, \alpha_{r}$ is changed by the algebraic independence over $\mathbb{Q}$. Below we will give a new direct proof of Theorem 1 which does not use the modified Cramér-Wald criterion (a statement of the type of Lemma 9 in [5]).

We note that Theorem 3 of [5] is true. In the case of rational $\lambda$, the Dirichlet series for $L(\lambda, \alpha, s)$ is reduced to ordinary Dirichlet series (with exponents $\log m$ ), therefore, even in the multidimensional case, we can use a limit theorem on the torus

$$
\hat{\Omega}=\prod_{p} \gamma_{p}
$$

where $\gamma_{p}=\gamma$ for all primes $p$, and the linear independence over $\mathbb{Q}$ of logarithms of prime numbers. The further proof runs in a standard way.

The same changes must be done also in Theorems 1 and 2 from [6], on the joint universality and functional independence of Lerch zeta-functions, since their 
proof is based on Theorem 2 of [5]. Thus, in Theorems 1 and 2 of [6] the condition that $\alpha_{1}, \ldots, \alpha_{r}$ are transcendental must be changed by a more strong requirement that the numbers $\alpha_{1}, \ldots, \alpha_{r}$ are algebraically independent over $\mathbb{Q}$.

We also note that the same changes must be done in Theorems 5.3.1, 5.3.2, 6.3.1 and 7.2.1 of [4].

For example, the transcendental numbers $\alpha_{1}=e^{-1}$ and $\alpha_{2}=e^{-2}$ are not algebraically independent over $\mathbb{Q}$, therefore they do not satisfy the hypotheses of Theorem 1. On the other hand, it is known that the numbers $e^{\pi}$ and $\pi$ are algebraically independent over $\mathbb{Q}$. So, we can take, for example, $\alpha_{1}=e^{\pi} / 12$ and $\alpha_{2}=\pi / 4$ in Theorem 1 .

Now we state one generalization of Theorems 1 and 2 (after the above correction) from [6]. Let $\lambda_{1}, \ldots, \lambda_{r}$ be arbitrary rational numbers with denominators $q_{1}, \ldots, q_{r}$, respectively. Denote by $k=\left[q_{1}, \ldots, q_{r}\right]$ the least common multiple, and define

$$
A=\left(\begin{array}{cccc}
e^{2 \pi i \lambda_{1}} & e^{2 \pi i \lambda_{2}} & \cdots & e^{2 \pi i \lambda_{r}} \\
e^{4 \pi i \lambda_{1}} & e^{4 \pi i \lambda_{2}} & \cdots & e^{4 \pi i \lambda_{r}} \\
\cdots & \cdots & \cdots & \cdots \\
e^{2 \pi k i \lambda_{1}} & e^{2 \pi k i \lambda_{2}} & \cdots & e^{2 \pi k i \lambda_{r}}
\end{array}\right)
$$

THEOREM 2. Suppose that $\alpha_{1}, \ldots, \alpha_{r}$ are algebraically independent over $\mathbb{Q}$ and that $\operatorname{rank}(A)=r$. Let $K_{j}$ be a compact subset of the strip $D_{0}=\left\{s \in \mathbb{C}: \frac{1}{2}<\sigma<1\right\}$ with connected complement, and let $f_{j}(s)$ be a continuous on $K_{j}$ function which is analytic in the interior of $K_{j}, j=1, \ldots, r$. Then, for every $\varepsilon>0$

$$
\liminf _{T \rightarrow \infty} \nu_{T}^{\tau}\left(\sup _{1 \leqslant j \leqslant r} \sup _{s \in K_{j}}\left|L\left(\lambda_{j}, \alpha_{j}, s+i \tau\right)-f_{j}(s)\right|<\varepsilon\right)>0 .
$$

THEOREM 3. Suppose that $\alpha_{1}, \ldots, \alpha_{r}$ are algebraically independent over $\mathbb{Q}$ and that $\operatorname{rank}(A)=r$. Let $F_{j}$ be a continuous on $\mathbb{C}^{N r}$ function, $j=0, \ldots, l$, and

$$
\begin{gathered}
\sum_{j=0}^{l} s^{j} F_{j}\left(L\left(\lambda_{1}, \alpha_{1}, s\right), \ldots, L\left(\lambda_{r}, \alpha_{r}, s\right), L^{\prime}\left(\lambda_{1}, \alpha_{1}, s\right), \ldots,\right. \\
\left.L^{\prime}\left(\lambda_{r}, \alpha_{r}, s\right), \ldots, L^{(N-1)}\left(\lambda_{1}, \alpha_{1}, s\right), \ldots, L^{(N-1)}\left(\lambda_{r}, \alpha_{r}, s\right)\right)=0
\end{gathered}
$$

identically for $s \in \mathbb{C}$. Then $F_{j} \equiv 0, j=0, \ldots, l$.

Theorem 3 is deduced from Theorem 2 in the same way as in [6] where Theorem 2 of [6] is obtained from Theorem 1 of [6].

\section{PROOF OF THEOREM 1}

2.1. Joint limit theorems for Dirichlet polynomials. First we will prove a joint limit theorem on the torus $\Omega^{r}$ for the probability measure

$Q_{T, r}(A)=\nu_{T}^{\tau}\left(\left(\left(\left(m+\alpha_{1}\right)^{i \tau}: m \in \mathbb{N}_{0}\right), \ldots,\left(\left(m+\alpha_{r}\right)^{i \tau}: m \in \mathbb{N}_{0}\right)\right) \in A\right), \quad A \in \mathcal{B}\left(\Omega^{r}\right)$. 
LEMMA 4. The probability measure $Q_{T, r}$ converges weakly to the Haar measure $m_{H r}$ on $\left(\Omega^{r}, \mathcal{B}\left(\Omega^{r}\right)\right)$ as $T \rightarrow \infty$.

Proof. The dual group of $\Omega^{r}$ is

$$
\bigoplus_{j=1}^{r} \bigoplus_{m=0}^{\infty} \mathbb{Z}_{m j}
$$

where $\mathbb{Z}_{m j}=\mathbb{Z}$ for all $m \in \mathbb{N}_{0}$ and $j=1, \ldots, r$. The element $\left(\underline{k}_{1}, \ldots, \underline{k}_{r}\right)=$ $\left(k_{01}, k_{11}, \ldots, k_{0 r}, k_{1 r}, \ldots\right) \in \bigoplus_{j=1}^{r} \bigoplus_{m=0}^{\infty} \mathbb{Z}_{m j}$, where only a finite number of integers $k_{m j}, m \in \mathbb{N}_{0}, j=1, \ldots, r$, are distinct from zero, acts on $\Omega^{r}$ by

$$
\left(\underline{x}_{1}, \ldots, \underline{x}_{r}\right) \rightarrow\left(\underline{x}_{1}^{\underline{k}_{1}}, \ldots, \underline{x}_{r}^{\underline{k}}\right)=\prod_{j=1}^{r} \prod_{m=0}^{\infty} x_{m j}^{k_{m j}}, \quad x_{j}=\left(x_{1 j}, x_{2 j}, \ldots\right), x_{m j} \in \gamma, m \in \mathbb{N}_{0}, j=1, \ldots, r .
$$

Therefore, the Fourier transform $g_{T, r}\left(\underline{k}_{1}, \ldots, \underline{k}_{r}\right)$ of the measure $Q_{T, r}$ is

$$
\begin{gathered}
g_{T, r}\left(\underline{k}_{1}, \ldots, \underline{k}_{r}\right)=\int_{\Omega^{r}} \prod_{j=1}^{r} \prod_{m=0}^{\infty} x_{m j}^{k_{m j}} \mathrm{~d} Q_{T, r}= \\
=\frac{1}{T} \int_{0}^{T} \prod_{j=1}^{r} \prod_{m=0}^{\infty} \mathrm{e}^{i \tau k_{m j} \log \left(m+\alpha_{j}\right)} \mathrm{d} \tau= \\
=\frac{1}{T} \int_{0}^{T} \exp \left\{i \tau \sum_{j=1}^{r} \sum_{m=1}^{\infty} k_{m j} \log \left(m+\alpha_{j}\right)\right\} \mathrm{d} \tau .
\end{gathered}
$$

Since $\alpha_{1}, \ldots, \alpha_{r}$ are algebraically independent over $\mathbb{Q}$, we find that

$$
g_{T, r}\left(\underline{k}_{1}, \ldots, \underline{k}_{r}\right)=\left\{\begin{array}{l}
1 \text { if }\left(\underline{k}_{1}, \ldots, \underline{k}_{r}\right)=(\underline{0}, \ldots, \underline{0}), \\
\frac{\exp \left\{i T \sum_{j=1}^{r} \sum_{m=0}^{\infty} k_{m j} \log \left(m+\alpha_{j}\right)\right\}-1}{i T \sum_{j=1}^{r} \sum_{m=0}^{\infty} k_{m j} \log \left(m+\alpha_{j}\right)} \text { if }\left(\underline{k}_{1}, \ldots, \underline{k}_{r}\right) \neq(\underline{0}, \ldots, \underline{0}) .
\end{array}\right.
$$

Consequently, we have

$$
\lim _{T \rightarrow \infty} g_{T, r}\left(\underline{k}_{1}, \ldots, \underline{k}_{r}\right)=\left\{\begin{array}{l}
1 \text { if }\left(\underline{k}_{1}, \ldots, \underline{k}_{r}\right)=(\underline{0}, \ldots, \underline{0}) \\
0 \text { if }\left(\underline{k}_{1}, \ldots, \underline{k}_{r}\right) \neq(\underline{0}, \ldots, \underline{0})
\end{array} .\right.
$$

Hence by continuity theorems for probability measures on locally compact groups ([2], Theorem 1.4.2), we obtain that the measure $Q_{T, r}$ converges weakly to $m_{H r}$ as $T \rightarrow \infty$. 
Now let $\sigma_{1 j}>\frac{1}{2}$, and, for $m, n \in \mathbb{N}_{0}$,

$$
v_{j}(m, n)=\exp \left\{-\left(\frac{m+\alpha_{j}}{n+\alpha_{j}}\right)^{\sigma_{1 j}}\right\}, \quad j=1, \ldots, r .
$$

Define, for $N_{j} \in \mathbb{N}_{0}, \hat{\omega}_{j} \in \Omega, s \in D$

$$
\begin{gathered}
L_{N_{j}, j, n}\left(\lambda_{j}, \alpha_{j}, s\right)=\sum_{m=0}^{N_{j}} \frac{\mathrm{e}^{2 \pi i \lambda_{j} m} v_{j}(m, n)}{\left(m+\alpha_{j}\right)^{s}}, \\
L_{N_{j}, j, n}\left(\lambda_{j}, \alpha_{j}, s, \hat{\omega}_{j}\right)=\sum_{m=0}^{N_{j}} \frac{\mathrm{e}^{2 \pi i \lambda_{j} m} v_{j}(m, n) \hat{\omega}_{j}(m)}{\left(m+\alpha_{j}\right)^{s}}, j=1, \ldots, r,
\end{gathered}
$$

and consider the weak convergence of the probability measures

$$
P_{T, N_{1}, \ldots, N_{r}, n}(A)=\nu_{T}^{\tau}\left(\left(L_{N_{1}, 1, n}\left(\lambda_{1}, \alpha_{1}, s+i \tau\right), \ldots, L_{N_{r}, r, n}\left(\lambda_{r}, \alpha_{r}, s+i \tau\right)\right) \in A\right)
$$

and

$\hat{P}_{T, N_{1}, \ldots, N_{r}, n}(A)=\nu_{T}^{\tau}\left(\left(L_{N_{1}, 1, n}\left(\lambda_{1}, \alpha_{1}, s+i \tau, \hat{\omega}_{1}\right), \ldots, L_{N_{r}, r, n}\left(\lambda_{r}, \alpha_{r}, s+i \tau, \hat{\omega}_{r}\right)\right) \in A\right)$, where $A \in \mathcal{B}\left(H^{r}(D)\right)$.

THEOREM 5. The probability measures $P_{T, N_{1}, \ldots, N_{r}, n}$ and $\hat{P}_{T, N_{1}, \ldots, N_{r}, n}$ both converge weakly to the same probability measure on $\left(H^{r}(D), \mathcal{B}\left(H^{r}(D)\right)\right)$ as $T \rightarrow$ $\infty$.

Proof. Let a function $h: \Omega^{r} \rightarrow H^{r}(D)$ be defined by

$$
h\left(\omega_{1}, \ldots, \omega_{r}\right)=\left(\sum_{m=0}^{N_{1}} \frac{\mathrm{e}^{2 \pi i \lambda_{1} m} v_{1}(m, n) \omega_{1}^{-1}(m)}{\left(m+\alpha_{1}\right)^{s}}, \ldots, \sum_{m=0}^{N_{r}} \frac{\mathrm{e}^{2 \pi i \lambda_{r} m} v_{r}(m, n) \omega_{r}^{-1}(m)}{\left(m+\alpha_{r}\right)^{s}}\right),
$$

$\left(\omega_{1}, \ldots, \omega_{r}\right) \in \Omega^{r}$. Then the function $h$ is continuous, and

$$
\begin{aligned}
& h\left(\left(\left(m+\alpha_{1}\right)^{i \tau}: m \in \mathbb{N}_{0}\right), \ldots,\left(\left(m+\alpha_{r}\right)^{i \tau}: m \in \mathbb{N}_{0}\right)\right) \\
= & \left(\left(L_{N_{1}, 1, n}\left(\lambda_{1}, \alpha_{1}, s+i \tau\right), \ldots, L_{N_{r}, r, n}\left(\lambda_{r}, \alpha_{r}, s+i \tau\right)\right) .\right.
\end{aligned}
$$

Hence $P_{T, N_{1}, \ldots, N, n}=Q_{T, r} h^{-1}$, therefore by Lemma 4 and Theorem 5.1 of [1] we obtain that the measure $P_{T, N_{1}, \ldots, N_{r}, n}$ converges weakly to $m_{H r} h^{-1}$ as $T \rightarrow \infty$.

Now let $h_{1}: \Omega^{r} \rightarrow \Omega^{r}$ be given by

$$
h_{1}\left(\omega_{1}, \ldots, \omega_{r}\right)=\left(\omega_{1} \hat{\omega}_{1}^{-1}, \ldots, \omega_{r} \hat{\omega}_{r}^{-1}\right) .
$$

Then

$$
\left(L_{N_{1}, 1, n}\left(\lambda_{1}, \alpha_{1}, s+i \tau, \hat{\omega}_{1}\right), \ldots, L_{N_{r}, r, n}\left(\lambda_{r}, \alpha_{r}, s+i \tau, \hat{\omega}_{r}\right)\right)=
$$




$$
=h\left(h_{1}\left(\left(\left(m+\alpha_{1}\right)^{i \tau}: m \in \mathbb{N}_{0}\right), \ldots,\left(\left(m+\alpha_{r}\right)^{i \tau}: m \in \mathbb{N}_{0}\right)\right)\right) .
$$

Similarly as above we find that $\hat{P}_{T, N_{1}, \ldots, N_{r}, n}$ converges weakly to the measure $m_{H r}\left(h h_{1}\right)^{-1}$ as $T \rightarrow \infty$. However, the invariance of the measure $m_{H r}$ yields

$$
m_{H r}\left(h h_{1}\right)^{-1}=\left(m_{H r} h_{1}^{-1}\right) h^{-1}=m_{H r} h^{-1},
$$

and the theorem follows.

2.2. Limit theorems for absolutely convergent series. For $j=1, \ldots, r$, let $\omega_{j} \in \Omega$, and

$$
L_{n, j}\left(\lambda_{j}, \alpha_{j}, s\right)=\sum_{m=0}^{\infty} \frac{\mathrm{e}^{2 \pi i \lambda_{j} m} v_{j}(m, n)}{\left(m+\alpha_{j}\right)^{s}}
$$

and

$$
L_{n, j}\left(\lambda_{j}, \alpha_{j}, s, \omega_{j}\right)=\sum_{m=0}^{\infty} \frac{\mathrm{e}^{2 \pi i \lambda_{j} m} \omega_{j}(m) v_{j}(m, n)}{\left(m+\alpha_{j}\right)^{s}} .
$$

Note that these two series both converge absolutely for $\sigma>\frac{1}{2}$. Define the probability measures

$$
P_{T, n}(A)=\nu_{T}^{\tau}\left(\left(L_{n, 1}\left(\lambda_{1}, \alpha_{1}, s+i \tau\right), \ldots, L_{n, r}\left(\lambda_{r}, \alpha_{r}, s+i \tau\right)\right) \in A\right)
$$

and

$$
\hat{P}_{T, n}(A)=\nu_{T}^{\tau}\left(\left(L_{n, 1}\left(\lambda_{1}, \alpha_{1}, s+i \tau, \omega_{1}\right), \ldots, L_{n, r}\left(\lambda_{r}, \alpha_{r}, s+i \tau, \omega_{r}\right)\right) \in A\right),
$$

where $A \in \mathcal{B}\left(H^{r}(D)\right)$.

TheOREM 6. On $\left(H^{r}(D), \mathcal{B}\left(H^{r}(D)\right)\right)$ there exists a probability measure $P_{n}$ such that the measures $P_{T, n}$ and $\hat{P}_{T, n}$ both converge weakly to $P_{n}$ as $T \rightarrow \infty$.

Proof. We apply Theorem 5 with $N_{1}=\ldots=N_{r} \stackrel{\text { def }}{=} N$. Then by Theorem 5 the measures $P_{T, N_{1}, \ldots, N_{r}, n} \stackrel{\text { def }}{=} P_{T, N, n}$ and $\hat{P}_{T, N_{1}, \ldots, N_{r}, n} \stackrel{\text { def }}{=} \hat{P}_{T, N, n}$ both converge to the same measure $P_{N, n}$ as $T \rightarrow \infty$.

First we observe that, for any fixed $n$, the family $\left\{P_{N, n}: N \in \mathbb{N}_{0}\right\}$ is tight. Let $\eta$ be a random variable defined on a certain probability space $(\hat{\Omega}, \mathcal{B}(\hat{\Omega}), \mathbb{P})$ and uniformly distributed on $[0,1]$. Define, for $j=1, \ldots, r$,

$$
X_{T, N, j, n}=X_{T, N, j, n}(s)=L_{N, j, n}\left(\lambda_{j}, \alpha_{j}, s+i T \eta\right),
$$

which is an $H(D)$ - valued random element defined on $(\hat{\Omega}, \mathcal{B}(\hat{\Omega}), \mathbb{P})$. Then by Theorem 5

$$
\underline{X}_{T, N, n} \stackrel{\text { def }}{=}\left(X_{T, N, 1, n}, \ldots, X_{T, N, r, n}\right) \underset{T \rightarrow \infty}{\stackrel{\mathcal{D}}{\longrightarrow}} \underline{X}_{N, n}
$$

where $\underline{X}_{N, n}=\left(X_{N, 1, n}, \ldots, X_{N, r, n}\right)$ is an $H^{r}(D)$-valued random element with the distribution $P_{N, n}$, and $\stackrel{\mathcal{D}}{\longrightarrow}$ means the convergence in distribution. 
For the investigation of the weak convergence of the measures $P_{T, n}$ and $\hat{P}_{T, n}$ we need a metric on $H^{r}(D)$ which induces its topology. Let $\left\{K_{n}\right\}$ be a sequence of compact subsets of $D$ such that $\bigcup_{n=1}^{\infty} K_{n}=D, K_{n} \subset K_{n+1}$, and if $K$ is a compact of $D$, then $K \subseteq K_{n}$ for some $n$. Then

$$
\begin{gathered}
\rho(f, g)=\sum_{n=1}^{\infty} 2^{-n} \frac{\rho_{n}(f, g)}{1+\rho_{n}(f, g)}, \quad f, g \in H(D), \\
\rho_{n}(f, g)=\sup _{s \in K_{n}}|f(s)-g(s)|,
\end{gathered}
$$

is a metric on $H(D)$ which induces its topology. Then

$$
\rho_{r}(\underline{f}, \underline{g})=\max _{1 \leqslant j \leqslant r} \rho\left(f_{j}, g_{j}\right),
$$

where $\underline{f}=\left(f_{1}, \ldots, f_{n}\right) \in H^{r}(D), \underline{g}=\left(g_{1}, \ldots, g_{n}\right) \in H^{r}(D)$, is a desired metric on $H^{r}(D)$.

The series for $L_{n, j}\left(\lambda_{j}, \alpha_{j}, s\right), j=1, \ldots, r$, converge absolutely for $\sigma>\frac{1}{2}$. Therefore, for $M_{l j}>0, j=1, \ldots, r, l \in \mathbb{N}$, and some $\sigma_{l}>1 / 2$

$$
\begin{gathered}
\limsup _{T \rightarrow \infty} \mathbb{P}\left(\sup _{s \in K_{l}}\left|X_{T, N, j, n}(s)\right|>M_{l j} \quad \text { for at least one } j=1, \ldots, r\right) \leqslant \\
\leqslant \sum_{j=1}^{r} \limsup _{T \rightarrow \infty} \mathbb{P}\left(\sup _{s \in K_{l}}\left|X_{T, N, j, n}(s)\right|>M_{l j}\right) \leqslant \\
\leqslant \sum_{j=1}^{r} \frac{1}{M_{l j}} \sup _{N \geqslant 0} \limsup _{T \rightarrow \infty} \frac{1}{T} \int_{0}^{T} \sup _{s \in K_{l}}\left|L_{N, j, n}\left(\lambda_{j}, \alpha_{j}, s+i \tau\right)\right| \mathrm{d} \tau \ll l \\
\ll \sum_{j=1}^{r} \frac{1}{M_{l j}} \sup _{N \geqslant 0} \limsup _{T \rightarrow \infty} \frac{1}{T} \int_{0}^{2 T}\left|L_{N, j, n}\left(\lambda_{j}, \alpha_{j}, \sigma_{l}+i t\right)\right| d t \ll \ll_{l} \\
\ll \sum_{j=1}^{r} \frac{1}{M_{l j}} \sup _{N \geqslant 0} \limsup _{T \rightarrow \infty}\left(\frac{1}{2 T} \int_{0}^{2 T}\left|L_{N, j, n}\left(\lambda_{j}, \alpha_{j}, \sigma_{l}+i t\right)\right|^{2} d t\right)^{1 / 2} \\
\ll \sum_{j=1}^{r} \frac{1}{M_{l j}} \sup _{N \geqslant 0}\left(\sum_{m=0}^{N} \frac{v_{j}^{2}(m, n)}{\left(m+\alpha_{j}\right)^{2 \sigma_{l}}}\right)^{1 / 2} \leqslant \\
\leqslant C_{l} \sum_{j=1}^{r} \frac{1}{M_{l j}}\left(\sum_{m=0}^{\infty} \frac{1}{\left(m+\alpha_{j}\right)^{2 \sigma_{l}}}\right)^{1 / 2} \stackrel{d e f}{=} C_{l} \sum_{j=1}^{r} \frac{R_{l j}}{M_{l j}}
\end{gathered}
$$


with a certain $C_{l}>0$ and

$$
R_{l j}=\left(\sum_{m=0}^{\infty} \frac{1}{\left(m+\alpha_{j}\right)^{2 \sigma_{l}}}\right)^{1 / 2}<\infty .
$$

Taking $M_{l j}=C_{l} R_{l j} 2^{l} r / \varepsilon$, hence we find that

$$
\limsup _{T \rightarrow \infty} \mathbb{P}\left(\sup _{s \in K_{l}}\left|X_{T, N, j, n}(s)\right|>M_{l j} \quad \text { for at least one } j=1, \ldots, r\right) \leqslant \frac{\varepsilon}{2^{l}} .
$$

Now (1) and (2) show that, for all $l \in \mathbb{N}$,

$$
\mathbb{P}\left(\sup _{s \in K_{l}}\left|X_{N, j, n}(s)\right|>M_{l j} \quad \text { for at least one } j=1, \ldots, r\right) \leqslant \frac{\varepsilon}{2^{l}} .
$$

Define

$$
H_{\varepsilon}^{r}=\left\{\left(f_{1}, \ldots, f_{r}\right) \in H^{r}(D): \sup _{s \in K_{l}}\left|f_{j}(s)\right| \leqslant M_{l j}, j=1, \ldots, r, l \in \mathbb{N}\right\} .
$$

Then the set $H_{\varepsilon}^{r}$ is compact, and by (3)

$$
\mathbb{P}\left(\underline{X}_{N, n}(s) \in H_{\varepsilon}^{r}\right) \geqslant 1-\varepsilon,
$$

or, by the definition of $\underline{X}_{N, n}$,

$$
P_{N, n}\left(H_{\varepsilon}^{r}\right) \geqslant 1-\varepsilon
$$

for all $N \in \mathbb{N}_{0}$. This proves the tightness of the family $\left\{P_{N, n}: N \in \mathbb{N}_{0}\right\}$.

By the definition, for $j=1, \ldots, r$,

$$
\lim _{N \rightarrow \infty} L_{N, j, n}\left(\lambda_{j}, \alpha_{j}, s\right)=L_{n, j}\left(\lambda_{j}, \alpha_{j}, s\right),
$$

the convergence being uniform on compact subsets of $D$. Therefore, for every $\varepsilon>$ 0 ,

$$
\begin{gathered}
\lim _{N \rightarrow \infty} \limsup _{T \rightarrow \infty} \nu_{T}^{\tau}\left(\rho_{r}\left(\underline{L}_{N, n}(s+i \tau), \underline{L}_{n}(s+i \tau)\right) \geqslant \varepsilon\right) \leqslant \\
\leqslant \lim _{N \rightarrow \infty} \limsup _{T \rightarrow \infty} \frac{1}{T \varepsilon} \int_{0}^{T} \rho_{r}\left(\underline{L}_{N, n}(s+i \tau), \underline{L}_{n}(s+i \tau)\right) d \tau \leqslant \\
\leqslant \lim _{N \rightarrow \infty} \limsup _{T \rightarrow \infty} \frac{1}{T \varepsilon} \sum_{j=1}^{r} \int_{0}^{T} \rho\left(L_{N, j, n}(s+i \tau), L_{n, j}(s+i \tau)\right) d \tau=0 .
\end{gathered}
$$

Here

$$
\underline{L}_{N, n}(s)=\left(L_{N, 1, n}\left(\lambda_{1}, \alpha_{1}, s\right), \ldots, L_{N, r, n}\left(\lambda_{r}, \alpha_{r}, s\right)\right)
$$


and

$$
\underline{L}_{n}(s)=\left(L_{n, 1}\left(\lambda_{1}, \alpha_{1}, s\right), \ldots, L_{n, r}\left(\lambda_{r}, \alpha_{r}, s\right)\right) .
$$

Now define, for $j=1, \ldots, r$,

$$
X_{T, j, n}=X_{T, j, n}(s)=L_{n, j}\left(\lambda_{j}, \alpha_{j}, s+i T \eta\right),
$$

and put

$$
\underline{X}_{T, n}=\left(X_{T, 1, n}, \ldots, X_{T, r, n}\right) .
$$

Then in view of (4)

$$
\lim _{N \rightarrow \infty} \limsup _{T \rightarrow \infty} \mathbb{P}\left(\rho_{r}\left(\underline{X}_{T, N, n}, \underline{X}_{T, n}\right) \geqslant \varepsilon\right)=0 .
$$

Since the family $\left\{P_{N, n}: N \in \mathbb{N}_{0}\right\}$ is tight, by the Prokhorov theorem it is relatively compact. Let $\left\{P_{N_{1}, n}\right\} \subset\left\{P_{N, n}\right\}$ be such that $P_{N_{1}, n}$ converges weakly to some measure $P_{n}$ as $N_{1} \rightarrow \infty$. Then we have that

$$
\underline{X}_{N_{1}, n} \underset{N_{1} \rightarrow \infty}{\stackrel{\mathcal{D}}{\longrightarrow}} P_{n}
$$

The space $H^{r}(D)$ is separable, and (1), (5) and (6) show that the hypothesis of Theorem 4.2 from [1] are satisfied. Consequently,

$$
\underline{X}_{T, n} \underset{T \rightarrow \infty}{\stackrel{\mathcal{D}}{\longrightarrow}} P_{n},
$$

and this is equivalent to the weak convergence of the measure $P_{T, n}$ to $P_{n}$ as $T \rightarrow$ $\infty$.

Formula (7) shows that the measure $P_{n}$ is independent of the sequence $N_{1}$. Therefore, we have

$$
\underline{X}_{N, n} \underset{N \rightarrow \infty}{\stackrel{\mathcal{D}}{\longrightarrow}} P_{n} .
$$

Now, repeating the above arguments for the random elements

$$
\underline{X}_{T, N, n}=\left(L_{N, 1, n}\left(\lambda_{1}, \alpha_{1}, s+i T \eta, \omega\right), \ldots, L_{N, r, n}\left(\lambda_{r}, \alpha_{r}, s+i T \eta, \omega\right)\right)
$$

and

$$
\underline{\widehat{X}}_{T, n}=\left(L_{n, 1}\left(\lambda_{1}, \alpha_{1}, s+i T \eta, \omega\right), \ldots, L_{n, r}\left(\lambda_{r}, \alpha_{r}, s+i T \eta, \omega\right)\right),
$$

and using (8) we similarly obtain that the measure $\widehat{P}_{T, n}$ also converges weakly to $P_{n}$ as $T \rightarrow \infty$. The theorem is proved.

2.3. Proof of Theorem 1. We start with approximation in mean of the vectors $\left(L\left(\lambda_{1}, \alpha_{1}, s\right), \ldots, L\left(\alpha_{1}, \lambda_{1}, s\right)\right)$ and $\left(L\left(\lambda_{1}, \alpha_{1}, s, \omega_{1}\right), \ldots, L\left(\lambda_{r}, \alpha_{r}, s, \omega_{r}\right)\right)$ by the vectors $\left(L_{n, 1}\left(\lambda_{1}, \alpha_{1}, s\right), \ldots, L_{n, r}\left(\lambda_{r}, \alpha_{r}, s,\right)\right)$ and $\left(L_{n, 1}\left(\lambda_{1}, \alpha_{1}, s, \omega_{1}\right), \ldots, L_{n, r}\left(\lambda_{r}, \alpha_{r}, s, \omega_{r}\right)\right)$, respectively. Let

$$
\begin{gathered}
\underline{L}(s)=\left(L\left(\lambda_{1}, \alpha_{1}, s\right), \ldots, L\left(\lambda_{r}, \alpha_{r}, s\right)\right), \\
\underline{L}(s, \underline{\omega})=\left(L\left(\lambda_{1}, \alpha_{1}, s, \omega_{1}\right), \ldots, L\left(\lambda_{r}, \alpha_{r}, s, \omega_{r}\right)\right)
\end{gathered}
$$

and

$$
\underline{L}_{n}(s, \underline{\omega})=\left(L_{n, 1}\left(\lambda_{1}, \alpha_{1}, s, \omega_{1}\right), \ldots, L_{n, r}\left(\lambda_{r}, \alpha_{r}, s, \omega_{r}\right)\right)
$$


LEMMA 7. We have

$$
\lim _{n \rightarrow \infty} \limsup _{T \rightarrow \infty} \frac{1}{T} \int_{0}^{T} \rho_{r}\left(\underline{L}(s+i \tau), \underline{L}_{n}(s+i \tau)\right) d \tau=0
$$

and, for almost all $\underline{\omega} \in \Omega^{r}$,

$$
\lim _{n \rightarrow \infty} \limsup _{T \rightarrow \infty} \frac{1}{T} \int_{0}^{T} \rho_{r}\left(\underline{L}(s+i \tau, \underline{\omega}), \underline{L}_{n}(s+i \tau, \underline{\omega})\right) d \tau=0 .
$$

Proof. Since $\alpha_{1}, \ldots, \alpha_{r}$ are algebraically independent over $\mathbb{Q}$, they are transcendental. Therefore, for each $j=1, \ldots, r$, Lemmas 5.2.11 and 5.2.13 of [4] imply

$$
\lim _{n \rightarrow \infty} \limsup _{T \rightarrow \infty} \frac{1}{T} \int_{0}^{T} \rho\left(L\left(\lambda_{j}, \alpha_{j}, s+i \tau\right), L_{n, j}\left(\lambda_{j}, \alpha_{j}, s+i \tau\right)\right) d \tau=0
$$

and, for almost all $\omega_{j} \in \Omega$,

$$
\lim _{n \rightarrow \infty} \limsup _{T \rightarrow \infty} \frac{1}{T} \int_{0}^{T} \rho\left(L\left(\lambda_{j}, \alpha_{j}, s+i \tau, \omega_{j}\right), L_{n, j}\left(\lambda_{j}, \alpha_{j}, s+i \tau, \omega_{j}\right)\right) d \tau=0 .
$$

From this and the definition of $\rho_{r}$ the lemma follows.

For $A \in \mathcal{B}\left(H^{r}(D)\right)$, define

$$
\widehat{P}_{T}(A)=\nu_{T}^{\tau}\left(\left(L\left(\lambda_{1}, \alpha_{1}, s+i \tau, \omega_{1}\right), \ldots, L\left(\lambda_{r}, \alpha_{r}, s+i \tau, \omega_{r}\right)\right) \in A\right) .
$$

THEOREM 8. On $\left(H^{r}(D), \mathcal{B}\left(H^{r}(D)\right)\right)$ there exists a probability measure $P$ such that the measures $P_{T}$ and $\widehat{P}_{T}$ (for almost all $\underline{\omega}$ ) both converge weakly to $P$ as $T \rightarrow \infty$.

Proof. We use the same way as that in the proof of Theorem 6. First we will show that the family of probability measures $\left\{P_{n}: n \in \mathbb{N}_{0}\right\}$ is tight.

By Theorem 6

$$
\underline{X}_{T, n} \underset{T \rightarrow \infty}{\stackrel{\mathcal{D}}{\longrightarrow}} \underline{X}_{n},
$$

where $\underline{X}_{n}=\left(X_{n, 1}, \ldots, X_{n, r}\right)$ is an $H^{r}(D)$ - valued random element with the distribution $P_{n}$.

Since $L_{n, j}\left(\lambda_{j}, \alpha_{j}, s\right)$ is convergent absolutely for $\sigma>1 / 2, j=1, \ldots, r$, we have

$$
\lim _{T \rightarrow \infty} \frac{1}{T} \int_{0}^{T}\left|L_{n, j}\left(\lambda_{j}, \alpha_{j}, \sigma+i t\right)\right|^{2} d t=\sum_{m=0}^{\infty} \frac{v_{j}^{2}(m, n)}{\left(m+\alpha_{j}\right)^{2 \sigma}} \leqslant \sum_{m=0}^{\infty} \frac{1}{\left(m+\alpha_{j}\right)^{2 \sigma}}
$$


uniformly in $n$. Hence it is not difficult to see that, for some $\sigma_{l}>1 / 2$,

$$
\begin{gathered}
\limsup _{T \rightarrow \infty} \frac{1}{T} \int_{0}^{T} \sup _{s \in K_{l}}\left|L_{n, j}\left(\lambda_{j}, \alpha_{j}, s+i \tau\right)\right| d \tau \leqslant C_{l} \limsup _{T \rightarrow \infty}\left(\frac{1}{2 T} \int_{0}^{2 T}\left|L_{n, j}\left(\lambda_{j}, \alpha_{j}, \sigma_{l}+i t\right)\right|^{2} d t\right)^{1 / 2} \\
\leqslant C_{l} R_{l j}<\infty
\end{gathered}
$$

with a certain constant $C_{l}>0$, where

$$
R_{l j}=\left(\sum_{m=0}^{\infty} \frac{1}{\left(m+\alpha_{j}\right)^{2 \sigma_{l}}}\right)^{1 / 2} .
$$

Therefore, for $M_{l j}=C_{l} R_{l j} 2^{l} r / \varepsilon, j=1, \ldots, r, l \in \mathbb{N}$,

$$
\limsup _{T \rightarrow \infty} \mathbb{P}\left(\sup _{s \in K_{l}}\left|X_{T, j, n}(s)\right|>M_{l j} \text { for at least one } j=1, \ldots, r\right) \leqslant \frac{\varepsilon}{2^{l}} \text {. }
$$

This and (9) imply

$$
\mathbb{P}\left(\sup _{s \in K_{l}}\left|X_{n, j}(s)\right|>M_{l j} \text { for at least one } j=1, \ldots, r\right) \leqslant \frac{\varepsilon}{2^{l}} .
$$

Hence we find that

$$
P_{n}\left(H_{\varepsilon}^{r}\right) \geqslant 1-\varepsilon
$$

for all $n \in \mathbb{N}_{0}$, that is the family $\left\{P_{n}: n \in \mathbb{N}_{0}\right\}$ is tight.

Now let, for $j=1, \ldots, r$,

$$
X_{T, j}=X_{T, j}(s)=L\left(\lambda_{j}, \alpha_{j}, s+i T \eta\right),
$$

and let

$$
\underline{X}_{T}=\left(X_{T, 1}, \ldots, X_{T, r}\right) .
$$

Then in view of Lemma 7 , for every $\varepsilon>0$,

$$
\begin{gathered}
\lim _{n \rightarrow \infty} \limsup _{T \rightarrow \infty} \nu_{T}^{\tau}\left(\rho_{r}\left(\underline{L}(s+i \tau), \underline{L}_{n}(s+i \tau)\right) \geqslant \varepsilon\right) \leqslant \\
\leqslant \frac{1}{\varepsilon T} \int_{0}^{T} \rho_{r}\left(\underline{L}(s+i \tau), \underline{L}_{n}(s+i \tau)\right) d \tau=0 .
\end{gathered}
$$

Hence

$$
\lim _{n \rightarrow \infty} \limsup _{T \rightarrow \infty} \mathbb{P}\left(\rho_{r}\left(\underline{X}_{T}(s), \underline{X}_{T, n}(s)\right) \geqslant \varepsilon\right)=0 .
$$

The family $\left\{P_{n}: n \in \mathbb{N}_{0}\right\}$ is relatively compact. Let $\left\{P_{n_{1}}\right\} \subset\left\{P_{n}\right\}$ be such that $P_{n_{1}}$ converges weakly to some measure $P$ on $\left(H^{r}(D), \mathcal{B}\left(H^{r}(D)\right)\right)$ as $n_{1} \rightarrow \infty$. Then

$$
\underline{X}_{n_{1}} \underset{n_{1} \rightarrow \infty}{\stackrel{\mathcal{D}}{\longrightarrow}} P \text {. }
$$


This, (10), (9) and Theorem 4.2 of [1] show that

$$
\underline{X}_{T} \underset{T \rightarrow \infty}{\stackrel{\mathcal{D}}{\longrightarrow}} P,
$$

that is $P_{T}$ converges weakly to $P$ as $T \rightarrow \infty$.

By (11) the measure $P$ is independent on the sequence $n_{1}$. Therefore,

$$
\underline{X}_{n} \underset{n \rightarrow \infty}{\stackrel{\mathcal{D}}{\longrightarrow}} P .
$$

By the same way as above, using (12), we obtain that the measure $\widehat{P}_{T}$ for almost all $\underline{\omega}$ also converges weakly to $P$ as $T \rightarrow \infty$.

To identify the limit measure in Theorem 8 , we will apply some facts from ergodic theory.

Let $a_{\tau, j}=\left\{\left(m+\alpha_{j}\right)^{-i \tau}: m \in \mathbb{N}_{0}\right\}, \tau \in \mathbb{R}, j=1, \ldots, r$. Then $\left\{a_{\tau, j}: \tau \in \mathbb{R}\right\}$, for each $j=1, \ldots, r$, is a one - parameter group. Define the one - parameter family $\left\{\Phi_{\tau}: \tau \in \mathbb{R}\right\}=\left\{\varphi_{\tau, 1}, \ldots, \varphi_{\tau, r}: \tau \in \mathbb{R}\right\}$ of transformations on $\Omega^{r}$ by $\varphi_{\tau, j}\left(\omega_{j}\right)=$ $a_{\tau, j} \omega_{j}, \omega_{j} \in \Omega_{j}, j=1, \ldots, r$. Then we have a one - parameter group of measurable transformations on $\Omega^{r}$.

LEMMA 9. The one-parameter group $\left\{\Phi_{\tau}: \tau \in \mathbb{R}\right\}$ is ergodic.

Proof. Let $\chi: \Omega^{r} \rightarrow \gamma$ be a character. Then

$$
\chi(\underline{\omega})=\prod_{j=1}^{r} \prod_{m=0}^{\infty} \omega_{j}^{k_{m j}}(m), \quad \underline{\omega} \in \Omega^{r},
$$

where only a finite number of integers $k_{m j}$ are distinct from zero. Suppose that $\chi$ is a non - principal character. Then

$$
\chi\left(a_{\tau, 1}, \ldots, a_{\tau, r}\right)=\prod_{j=1}^{r} \prod_{m=0}^{\infty}\left(m+\alpha_{j}\right)^{-i \tau k_{m j}}=\exp \left\{-i \tau \sum_{j=1}^{r} \sum_{m=0}^{\infty} k_{m j} \log \left(m+\alpha_{j}\right)\right\},
$$

where only a finite number of integers $k_{m j} \neq 0$. Since $\alpha_{1}, \ldots, \alpha_{r}$ are algebraically independent, we have that there exists a $\tau_{0} \neq 0$ such that

$$
\chi\left(a_{\tau_{0}, 1}, \ldots, a_{\tau_{0}, r}\right) \neq 1 .
$$

The further proof runs in the same way as than in [3], Theorem 5.3.6.

Proof of Theorem 1. Let $A \in \mathcal{B}\left(H^{r}(D)\right)$ be a continuity set of the measure $P$ in Theorem 8 . Then by Theorem 2.1 of [1] and Theorem 8

$$
\lim _{T \rightarrow \infty} \nu_{T}^{\tau}\left(\left(L\left(\lambda_{1}, \alpha_{1}, s+i \tau, \omega_{1}\right), \ldots, L\left(\lambda_{r}, \alpha_{r}, s+i \tau, \omega_{r}\right)\right) \in A\right)=P(A)
$$

for almost all $\underline{\omega} \in \Omega^{r}$. Now we fix the set $A$ and define a random variable $\theta$ on the space $\left(\Omega^{r}, \mathcal{B}\left(\Omega^{r}\right), m_{H r}\right)$ by the formula

$$
\theta(\underline{\omega})=\left\{\begin{array}{lll}
1 & \text { if } & L(s, \underline{\omega}) \in A \\
0 & \text { if } & L(s, \underline{\omega}) \notin A .
\end{array}\right.
$$


Denote by $\mathbb{E}(\theta)$ the expectation of $\theta$. Then we have that

$$
\mathbb{E}(\theta)=\int_{\Omega^{r}} \theta d m_{H r}=m_{H r}\left(\underline{\omega} \in \Omega^{r}: L(s, \underline{\omega}) \in A\right)=P_{L}(A) .
$$

In virtue of Lemma 9 the random process $\theta\left(\Phi_{\tau}(\underline{\omega})\right)$ is ergodic. Therefore, by the Birkhoff-Khinchine theorem

$$
\lim _{T \rightarrow \infty} \frac{1}{T} \int_{0}^{T} \theta\left(\Phi_{\tau}(\underline{\omega})\right) d \tau=\mathbb{E}(\theta)
$$

for almost all $\underline{\omega} \in \Omega^{r}$. On the other hand, by the definition of $\theta$ and $\Phi_{\tau}$ we find

$$
\frac{1}{T} \int_{0}^{T} \theta\left(\Phi_{\tau}(\underline{\omega})\right) d \tau=\nu_{T}^{\tau}\left(\left(L\left(\lambda_{1}, \alpha_{1}, s+i \tau, \omega_{1}\right), \ldots, L\left(\lambda_{r}, \alpha_{r}, s+i \tau, \omega_{r}\right)\right) \in A\right) .
$$

This and (14), (15) show that

$$
\lim _{T \rightarrow \infty} \nu_{T}^{\tau}\left(\left(L\left(\lambda_{1}, \alpha_{1}, s+i \tau, \omega_{1}\right), \ldots, L\left(\lambda_{r}, \alpha_{r}, s+i \tau, \omega_{r}\right)\right) \in A\right)=P_{L}(A)
$$

for almost all $\underline{\omega} \in \Omega^{r}$. Hence, by $(13), P(A)=P_{L}(A)$ for any continuity set $A$ of the measure $P$, and this implies the equality $P(A)=P_{L}(A)$ for all $A \in$ $\mathcal{B}\left(H^{r}(D)\right)$. The theorem is proved.

\section{PROOF OF THEOREM 2.}

3.1. A limit theorem. The proof of Theorem 2 is based on a corollary of Theorem 1. Denote by $P_{\underline{L}_{0}}$ the restriction of the distribution $P_{\underline{L}}$ of the random element $\underline{L}(s, \underline{\omega})$ to $H^{r}\left(D_{0}\right)$.

COROLLARY 10. Suppose that $\alpha_{1}, \ldots, \alpha_{r}$ are algebraically independent over $\mathbb{Q}$. Then the probability measure

$$
\nu_{T}^{\tau}\left(\left(L\left(\lambda_{1}, \alpha_{1}, s+i \tau\right), \ldots, L\left(\lambda_{r}, \alpha_{r}, s+\tau\right)\right) \in A\right), \quad A \in \mathcal{B}\left(H^{r}\left(D_{0}\right)\right)
$$

converges weakly to $P_{\underline{L}_{0}}$ as $T \rightarrow \infty$.

Proof. The function $h: H(D) \rightarrow H^{r}\left(D_{0}\right)$ defined by $h(\underline{g})=\left.\underline{g}\right|_{s \in D_{0}}, \underline{g} \in$ $H^{r}(D)$, obviously, is continuous. Therefore, the corollary follows from Theorem 1 and Theorem 5.1 of [1].

3.2. The support of the measure $P_{\underline{L}_{0}}$. We recall that the support of the measure $P_{\underline{L}_{0}}$ is a minimal closed set $S_{P_{\underline{L}_{0}}} \subseteq H^{r}\left(D_{0}\right)$ such that $P_{S_{P_{\underline{L}_{0}}}}=1$. The set $S_{P_{\underline{L}_{0}}}$ consists of all $\underline{g} \in H^{r}(D)$ such that for every neighborhood $G$ of $\underline{g}$ the inequality $P_{\underline{L}_{0}}(G)>0$ holds. 
THEOREM 10. Suppose that $\alpha_{1}, \ldots, \alpha_{r}$ are algebraically independent over $\mathbb{Q}$ and $\operatorname{rank}(A)=r$. Then the support of $P_{\underline{L}_{0}}$ is the whole of $H^{r}\left(D_{0}\right)$.

Let

$$
\widehat{\underline{L}}_{0}(s, \omega)=\left(L\left(\lambda_{1}, \alpha_{1}, s, \omega\right), \ldots, L\left(\lambda_{r}, \alpha_{r}, s, \omega\right)\right), \quad s \in D_{0},
$$

where

$$
L\left(\lambda_{j}, \alpha_{j}, s, \omega\right)=\sum_{m=0}^{\infty} \frac{e^{2 \pi i m \lambda_{j}} \omega(m)}{\left(m+\alpha_{j}\right)^{s}},
$$

$\omega \in \Omega, j=1, \ldots, r$. Then, clearly, $S_{P_{\underline{L}_{0}}} \supseteq S_{P_{\widehat{L}_{0}}}$. Therefore, it suffices to prove that $S_{\widehat{P}_{\widehat{L}_{0}}}=H^{r}\left(D_{0}\right)$.

The support of each $\omega(m)$ is the unit circle $\gamma$. Thus, the support of

$$
\frac{e^{2 \pi i m \lambda_{j}} \omega(m)}{\left(m+\alpha_{j}\right)^{s}}
$$

is the set

$$
\left\{g \in H\left(D_{0}\right): g(s)=\frac{e^{2 \pi i m \lambda_{j}} a}{\left(m+\alpha_{j}\right)^{s}}, a \in \gamma\right\}, \quad m \in \mathbb{N}_{0}, j=1, \ldots, r .
$$

Since $\left\{\omega(m): m \in \mathbb{N}_{0}\right\}$ is a sequence of independent random variables defined on the probability space $\left(\Omega, \mathcal{B}(\Omega), m_{H}\right)$,

$$
\left\{\frac{e^{2 \pi i m \lambda_{j}} \omega(m)}{\left(m+\alpha_{j}\right)^{s}}: m \in \mathbb{N}_{0}\right\}, \quad j=1, \ldots, r,
$$

is a sequence of independent $H\left(D_{0}\right)$ - valued random elements defined on the above space. Therefore, by Lemma 5 of [7] the support $S_{{\underline{\underline{\underline{L}}_{0}}}}$ is the closure of the set of all convergent series

$$
\sum_{m=0}^{\infty}\left(\frac{e^{2 \pi i m \lambda_{1}} a_{m}}{\left(m+\alpha_{1}\right)^{s}}, \ldots, \frac{e^{2 \pi i m \lambda_{r}} a_{m}}{\left(m+\alpha_{r}\right)^{s}}\right)
$$

where $a_{m} \in \gamma$. Thus, we have to show that the latter set is dense in $H^{r}\left(D_{0}\right)$. For this we will use Lemma 6 of [7].

Let $\left\{b_{m}: b_{m} \in \gamma, m \in \mathbb{N}_{0}\right\}$ be a sequence such that the series

$$
\sum_{m=0}^{\infty}\left(\frac{e^{2 \pi i m \lambda_{1}} b_{m}}{\left(m+\alpha_{1}\right)^{s}}, \ldots, \frac{e^{2 \pi i m \lambda_{r}} b_{m}}{\left(m+\alpha_{r}\right)^{s}}\right)
$$

converges in $H^{r}\left(D_{0}\right)$. Such a sequence exists, since

$$
\sum_{m=0}^{\infty}\left(\frac{e^{2 \pi i m \lambda_{1}} \omega(m)}{\left(m+\alpha_{1}\right)^{s}}, \ldots, \frac{e^{2 \pi i m \lambda_{r}} \omega(m)}{\left(m+\alpha_{r}\right)^{s}}\right)
$$


is an $H^{r}\left(D_{0}\right)$ - valued random element. By the definition of $D_{0}$, for every compact subset $K$ of $D_{0}$,

$$
\sum_{m=0}^{\infty} \sum_{j=1}^{r} \sup _{s \in K} \frac{1}{\left(m+\alpha_{j}\right)^{2 \sigma}}<\infty .
$$

Therefore, it remains to verify only the hypothesis a) of Lemma 6 from [7]. Let $\mu_{1}, \ldots, \mu_{r}$ be complex measures on $(\mathbb{C}, \mathcal{B}(\mathbb{C}))$ with compact supports contained in $D_{0}$ and such that

$$
\sum_{m=0}^{\infty}\left|\sum_{j=1}^{r} \int_{\mathbb{C}} \frac{e^{2 \pi i m \lambda_{j}}}{\left(m+\alpha_{j}\right)^{s}} d \mu_{j}(s)\right|<\infty .
$$

Since (see $[6])$

$$
(m+\alpha)^{-s}=m^{-s}+O\left(m^{-1-\sigma}|s| e^{O(|s|)}\right),
$$

(17) and the properties of the measures $\mu_{1}, \ldots, \mu_{r}$ show that

$$
\sum_{m=0}^{\infty}\left|\sum_{j=1}^{r} \int_{\mathbb{C}} \frac{e^{2 \pi i m \lambda_{j}}}{m^{s}} d \mu_{j}(s)\right|<\infty .
$$

Hence, by the periodicity of $e^{2 \pi i m \lambda_{j}}$, for every $l=1, \ldots, k$,

$$
\sum_{\substack{m=0 \\ m=l(\bmod k)}}^{\infty}\left|\sum_{j=1}^{r} \int_{\mathbb{C}} \frac{e^{2 \pi i l \lambda_{j}}}{m^{s}} d \mu_{j}(s)\right|<\infty .
$$

Define

$$
\nu_{l}(A)=\sum_{j=1}^{r} e^{2 \pi i l \lambda_{j}} \mu_{j}(A), \quad A \in \mathcal{B}(\mathbb{C}), l=1, \ldots, k .
$$

Then, clearly, $\nu_{1}, \ldots, \nu_{l}$ are complex measures on $(\mathbb{C}, \mathcal{B}(\mathbb{C}))$ with compact supports obtained in $D_{0}$, and in view of (18)

$$
\sum_{\substack{m=0 \\ m \equiv l(\bmod k)}}^{\infty}\left|\int_{\mathbb{C}} m^{-s} d \nu_{l}(s)\right|<\infty, \quad l=1, \ldots, k .
$$

Now we put

$$
\rho_{l}(z)=\int_{\mathbb{C}} e^{-s z} d \nu_{l}(s), \quad z \in \mathbb{C}, \quad l=1, \ldots, k
$$

Then by (19)

$$
\sum_{\substack{m=0 \\ m \equiv l(\bmod k)}}^{\infty}\left|\rho_{l}(\log m)\right|<\infty, \quad l=1, \ldots, k .
$$


Clearly, $\rho_{l}(z)$ is an entire function of exponential type, $l=1, \ldots, k$. Thus, by Lemma 6.4 .10 of [3], either $\rho_{l} \equiv 0$, or

$$
\limsup _{x \rightarrow \infty} \frac{\log \left|\rho_{l}(x)\right|}{x}>-1, \quad l=1, \ldots, k
$$

Suppose that (21) is true. Then Lemma 5 of [6] (which is a version of the "positive density method") shows that

$$
\sum_{\substack{m=0 \\ m \equiv l(\bmod k)}}^{\infty}\left|\rho_{l}(\log m)\right|=\infty, \quad l=1, \ldots, k,
$$

and this contradicts (20). Therefore, we have that, for $l=1, \ldots, k, \rho_{l}(z) \equiv 0$. Hence, by the definition of the measures $\nu_{1}, \ldots, \nu_{l}$,

$$
\sum_{j=1}^{r} e^{2 \pi i l \lambda_{j}} \int_{\mathbb{C}} e^{-s z} d \mu_{j}(s) \equiv 0, \quad l=1, \ldots, k .
$$

Since $\operatorname{rank}(A)=r$, this implies

$$
\int_{\mathbb{C}} e^{-s z} d \mu_{j}(s) \equiv 0, \quad j=1, \ldots, r,
$$

and we easily deduce that

$$
\int_{\mathbb{C}} s^{l} d \mu_{j}(s) \equiv 0
$$

for all $j=1, \ldots, r$ and $l \in \mathbb{N}_{0}$. Hence we obtain that all hypotheses of Lemma 6 from [7] are satisfied, and we have that the set of all convergent series

$$
\sum_{m=0}^{\infty}\left(\frac{e^{2 \pi i m \lambda_{1}} b_{m} a_{m}}{\left(m+\alpha_{1}\right)^{s}}, \ldots, \frac{e^{2 \pi i m \lambda_{r}} b_{m} a_{m}}{\left(m+\alpha_{r}\right)^{s}}\right)
$$

with $a_{m} \in \gamma$ is dense in $H^{r}\left(D_{0}\right)$. Clearly, the set of all convergent series (16) also has the same property. The theorem is proved.

Proof of Theorem 2. The proof uses Theorem 10 and is the same that of Theorem 1 from [6].

\section{REFERENCES}

1. P. Billingsley, Convergence of Probability measures, John Wiley, New York (1968).

2. H. Heyer, Probability Measures on Locally Compact Groups, Springer - Verlag, Berlin (1977).

3. A. Laurinčikas, Limit Theorems for the Riemann Zeta-Function, Kluwer, Dordrecht (1996).

4. A. Laurinčikas, R. Garunkštis, The Lerch Zeta - Function, Kluwer, Dordrecht (2002). 
5. A. Laurinčikas, K. Matsumoto, Joint value - distribution theorems on Lerch zeta - functions, Lith. Math. J., 38(3), 238-249 (1998).

6. A. Laurinčikas, K. Matsumoto, The joint universality and the functional independence for Lerch zeta - functions, Nagoya Math. J., 157, 211-227 (2000).

7. A. Laurinčikas, K. Matsumoto, The joint universality of zeta - functions attached to certain cusp forms, Fiz. - mat. fak. moksl. seminaro darbai, 5, 58-75 (2002).

\section{Jungtinès reikšmiụ pasiskirstymo teoremos Lercho dzeta funkcijoms. II}

\section{A. Laurinčikas, K. Matsumoto.}

Pateikti ištaisyti kai kuriụ teoremụ iš [5] ir [6] formulavimai apie jungtinị Lercho dzeta funkcijụ reikšmiụ pasiskirstymạ (ribinès teoremos, universalumas, funkcinis nepriklausomumas). Be to, pateiktas naujas tiesioginis jungtinès ribinès teoremos analiziniụ funkcijụ erdvejje i̧rodymas bei praplèsta jungtinè universalmuo teorema. 